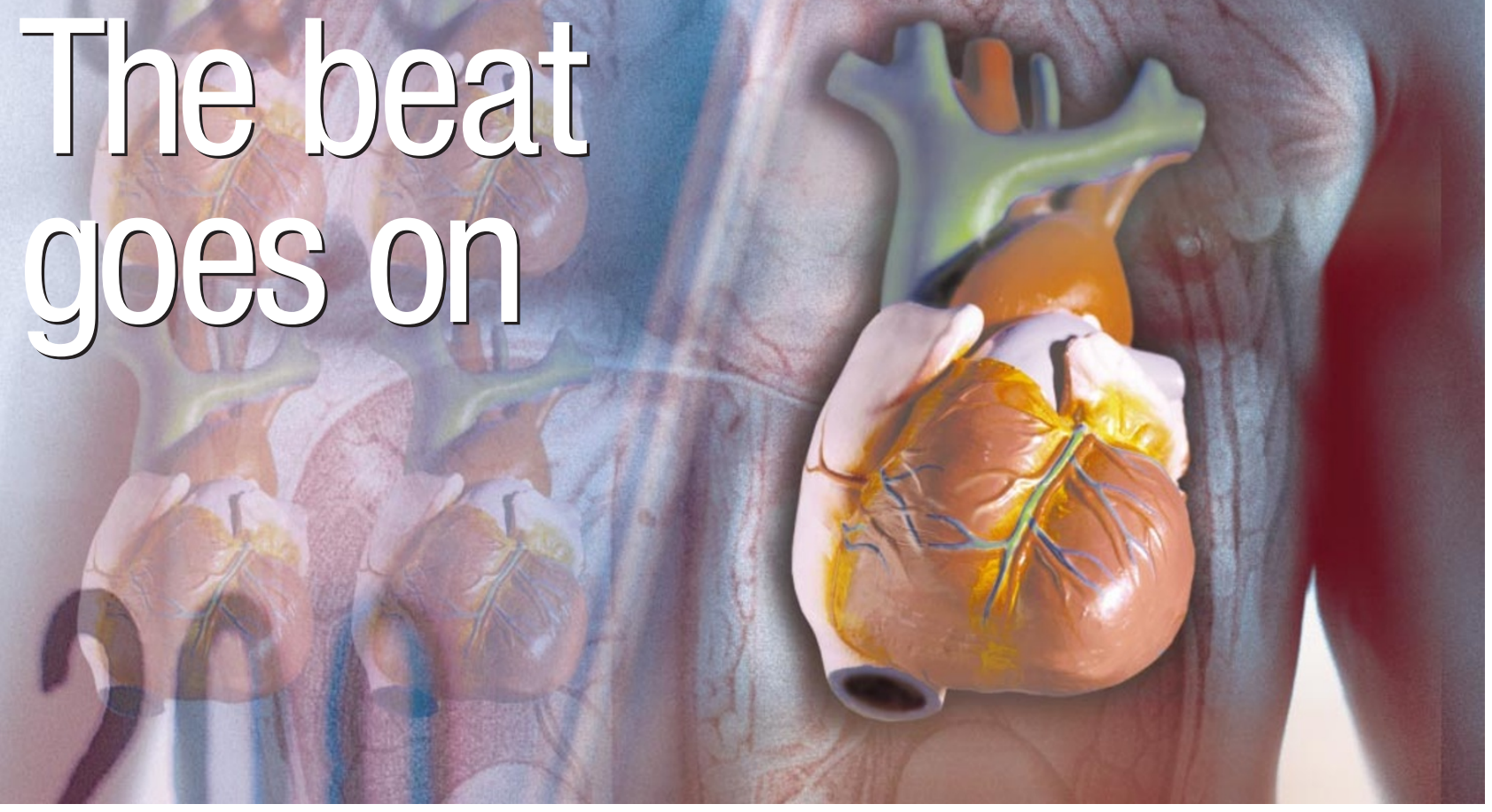

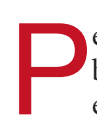

erhaps it was just millennial hubris, but in 1999 the world seemed full of enthusiasm for science and technology. The NASDAQ high-tech stock market was booming; the Human Genome Project was racing towards completion. And one group of scientists was talking up a plan to grow a fully functioning heart in the lab, starting with a culture of human cells in a petri dish. All they needed was ten years, plus about US\$5 billion, and our worries about the shortage of organ donors would be over.

The initiative, known as Living Implants from Engineering (LIFE), would rival the Human Genome Project in scale and in its potential to change medical practice. But four years later, LIFE has lost its lustre. The grand project remains largely unfunded, and functions mainly as a loose coalition of researchers who share their findings at meetings. "LIFE collapsed into the form of a club," admits its chief visionary, Michael Sefton of the University of Toronto in Canada.

Despite LIFE's stuttering start, tissue engineering retains enormous clinical promise. Tissue-engineered skin grafts have already won marketing approval from the US Food and Drug Administration, and implants of lab-grown cartilage are now in clinical trials. But these are relatively simple tissues. Growing organs as complex as the heart is an immensely greater challenge. "It would be wonderful if you could do it," says Robert Langer of the Massachusetts Institute of Technology (MIT) in Cambridge, who helped to pioneer the field of tissue engineering in the mid-1980s.

'If' remains the operative word. "We are all dreaming of this artificial heart beating in a dish, but I think this is unlikely to happen over the next few years," says Thomas Eschenhagen, a tissue engineer at Eppendorf University Hospital in Hamburg, Germany. "The heart is simply too complex."

\section{Growth area}

Faced with this daunting complexity, several research groups are instead working on the interim goal of growing some of the heart's component parts - patches of cardiac muscle, valves, coronary blood vessels and so on. One of the largest projects is the BioEngineered Autologous Tissue (BEAT) initiative. Led by Buddy Ratner of the University of Washington in Seattle, BEAT received a \$10-million, five-year grant in 2000 from the US National Institutes of Health to create patches of cardiac muscle to repair the damage caused by coronary heart attacks.

If that effort is successful, the BEAT team will try to create a complete ventricle, one of the muscular chambers that pump blood around the body. And in the long run, Ratner hasn't written off the idea of growing an entire heart from scratch. "The sheer audacity of the heart project keeps things exciting and stimulates us to think out of the box," he says.

There's also a pressing clinical need for heart tissue. Presently, those who require a transplant can end up waiting several years for a donated organ, and their problems don't end there: because of immunological rejection and other complications, donated hearts often last for only a decade or so.

The basics of tissue engineering are relatively straightforward: first build a scaffold on which tissue can grow, then seed some cells into it, and tuck it away in a specially designed 'bioreactor' that provides nutrients and oxygen. When the cells have multiplied to fill the scaffold, remove the structure from the bioreactor and implant it into the body. In 1995, the principle was demonstrated to the world in those famous images of a 'human' ear made from scaffold-grown cartilage protruding from the back of a mouse.

Unfortunately, heart tissue is rather more difficult to grow. Adult cardiac muscle cells tend not to divide, so the scaffold must be seeded with cells from another source. One idea is to use skeletal-muscle cells, which proliferate readily. Over the past few years, researchers have also become excited by the potential of stem cells extracted from adult bone marrow, or from few-day-old human embryos, both of which can differentiate into cardiac muscle cells if given the right stimuli. Indeed, it may be possible to repair small areas of damaged cardiac muscle by injecting stem cells or skeletal-muscle cells directly into the tissue - ongoing clinical trials involving patients' own skeletal-muscle cells have 


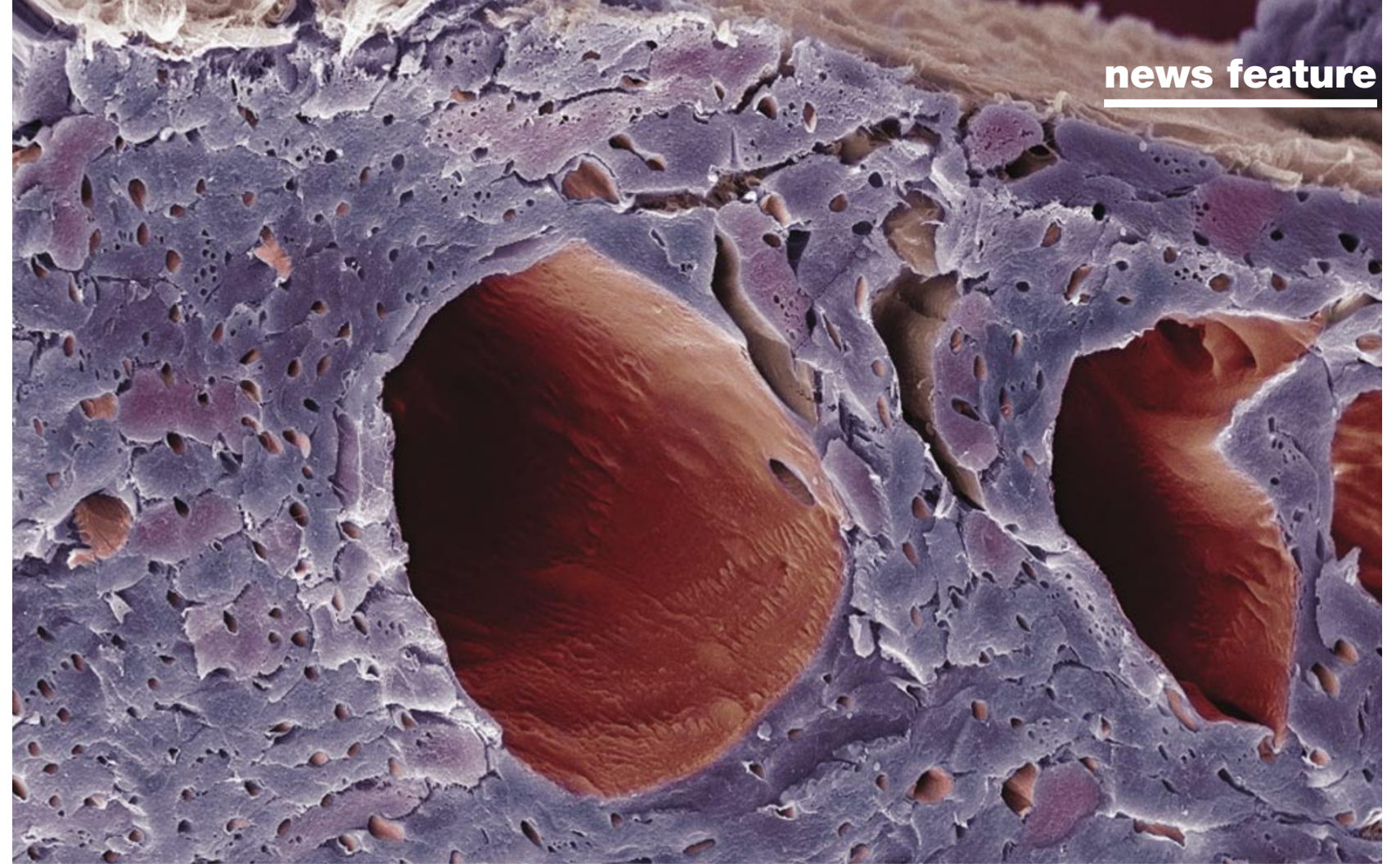

Heart of the matter: tissue engineers striving to grow replacements for natural cardiac muscle need to find ways to deliver nutrients to their tissues, much as capillaries (shown here in red) do in natural heart tissue.

yielded some improvement in muscle contraction. "We can use the heart itself as both a scaffold and bioreactor," says Charles Murry of the University of Washington, a cardiac pathologist and a member of the BEAT team.

Although direct injection of cells could successfully mend small areas of damage, repairing the large areas of dead tissue that result when a major coronary artery becomes blocked will require the lab-grown patches of muscle that are BEAT's main focus. To grow in three dimensions, cells need some sort of scaffold, which should be accepted by the body and ideally degrade over time into nontoxic components. The leading ideas involve using either biomolecules such as collagen, which provides mechanical support for natural tissues, or synthetic, biodegradable polymers such as poly(L)-lactic acid. To maximize the likelihood that seeded cells will 'take' to these scaffolds, some teams are coating their polymers with cell-adhesion proteins or designing them to release cell-signalling molecules ${ }^{1}$. Eventually, researchers hope to create scaffolds that encode specific instructions for controlling tissue formation, by analogy to the signals that control embryological development.

But there is still much to be done to perfect the mechanical properties of the scaffold. One of the most intriguing approaches is that of BEAT project member Kimberly Woodhouse of the University of
Toronto. Her group has designed a scaffold made of chains of elastin polypeptides, a biomolecule that provides elastic properties to some of our tissues. By experimenting with different molecules to crosslink the polypeptide chains, she can create structures that range from a delicate mesh to a material with the properties of an elastic band ${ }^{2}$. For a patch of heart muscle, the ideal scaffold would be something that combines the properties of both - allowing cells to burrow into the matrix but providing as much strength and elasticity as a beating heart.

\section{In thickness and in health}

Perhaps the biggest challenge, however, is working out how to grow three-dimensional structures that contain more than a few layers of muscle cells. Most bioreactors simply cannot supply enough nutrients and oxygen to the growing tissue. Whereas human heart muscle is up to 2 centimetres thick, growth in a bioreactor typically stops once the tissue is about 100 micrometres, or 4-7 cell layers, thick. Beyond this thickness, the innermost cells are too far from the supply of fresh growth medium to thrive.

Listen up: tissue engineering was unveiled to the world eight years ago, in the form of this famously eary mouse.
One approach is to sidestep the problem by abandoning scaffolds and growing single layers of cells that can then be sandwiched into slabs of tissue like a layered cake. Researchers led by Teruo Okano at the Tokyo Women's Medical University are attempting this feat using cardiac-muscle cells from newborn rats - these cells are still able to divide. Okano's team grows the cells on polymer surfaces that allow the intact cell layers to detach when the culture temperature is reduced ${ }^{3}$. In experiments reported last November at the Scientific Sessions of the American Heart Association (AHA) in Chicago, the researchers laid four of these sheets on top of each other until they fused. They then implanted them under the skin of immunodeficient rats. When the researchers opened the skin six months later, the engineered cardiac tissue was beating and blood vessels had permeated it.

Most researchers, however, are working on ways to simulate the presence of blood vessels in or around the scaffold to allow tissues to attain greater thicknesses. For instance, scientists led by Gordana Vunjak-Novakovic, a colleague of Langer at MIT, have designed a bioreactor that forces nutrient medium to perfuse directly through a cell-seeded scaffold instead of simply flowing around it. In new experiments using scaffolds containing cardiac-muscle cells from newborn rats, this bioreactor produced cardiac tissue with more evenly distributed cells and much higher cell densities than in any previous study ${ }^{4}$.

Eventually, it may be possible to grow tissues on scaffolds interweaved with artificial capillaries. At the Hope Heart Institute in Seattle, the BEAT project's Robert 
Vernon is designing one such artificial capillary bed. He spreads a dissolvable material out in thin, branch-like patterns, and then pours on a solution containing collagen and waits for it to solidify. Next, he uses a solvent to dissolve away the original material, leaving capillary-like tracks in the collagen. Vernon plans to sandwich sheets of this material between layers of cardiac muscle to create a three-dimensional block of tissue.

Vernon's vessels won't be able to carry blood because it would clot in the collagen tubes. But his artificial capillary beds could ferry cell-culture medium that could be crucial to keeping the tissue alive once it has grown. One of the challenges for the BEAT project will be in keeping the muscle patches oxygenated during implantation and for some weeks afterwards, until the patient's own vessels begin to colonize the implant. Vernon believes that his capillary bed could be hooked up to a miniature pump that would bathe the patch with nutrient fluid during this critical period.

Other researchers are experimenting with using the body itself as a bioreactor. In one such study, Jonathan Leor and his colleagues at Tel Aviv University's Sheba Medical Center in Israel seeded cells from embryonic and newborn rats onto threedimensional scaffolds, cultured them in a regular bioreactor for four days to assess their viability, and then surgically implanted the tissue in rats' body cavities. While the tissues were in their rat hosts, blood vessels grew into them. After a week, the researchers removed the tissue and used it to patch holes they had made in the hearts of another set of rats. Last November, Leor told the AHA's Scientific Sessions that the tissue patches seemed to integrate successfully into the rats' heart tissue. "By growing tissues in the body cavity, it is possible to create an environment that duplicates the conditions for the development of a normal tissue," he says.

Growing a patch of heart muscle is only half of the battle: ensuring that it can actually pump blood is far from trivial. The heart is a powerful muscle, and engineered tissue needs to be similarly vigorous. So some researchers are sending their muscles to the gym for a workout. In Hamburg, Eschenhagen and his colleagues are making cylindrical patches of cardiac muscle using cardiacmuscle cells from newborn rats as a startingpoint. But instead of seeding the cells on a scaffold, Eschenhagen mixes them into a collagen gel and casts them in a cylindrical mould. After a few days, he transfers the tissue patch to a stretching device that puts it through a workout that simulates the heart's contractions ${ }^{5}$. When Eschenhagen implanted the tissues into rats, that the cardiac cylinders contracted up to four times more vigorously than unstretched tissues ${ }^{6}$.

While Eschenhagen and others are per-

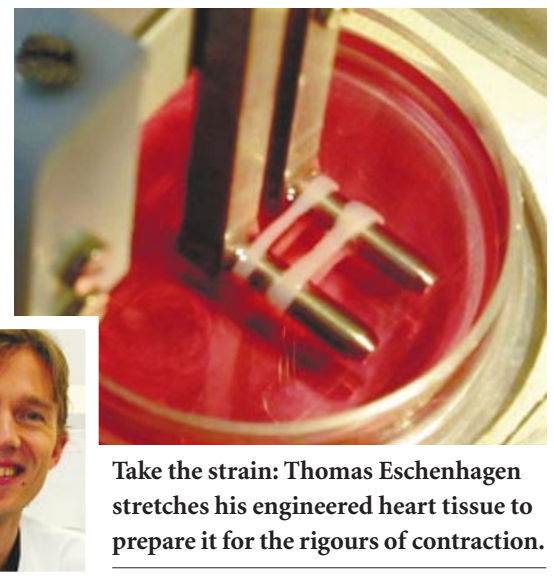

fecting their techniques for growing cardiac muscle, other groups are trying to engineer other components of the organ. Finding a way to create replacements for blocked coronary arteries is important, because not all patients who need bypass surgery have sufficiently healthy vessels to provide their own grafts. A team involving Langer, for instance, has grown functional arteries by seeding a tubular scaffold with muscle cells from a cow's aorta. Once this had grown to fill the scaffold, the researchers seeded the tube's interior with endothelial cells, which form the smooth lining of blood vessels. Throughout the procedure, fluid was pulsed through the tubes to simulate the mechanical stresses that blood vessels must face. When implanted into miniature pigs, these tissue-engineered arteries seemed to be functioning well up to 24 days later ${ }^{7}$.

\section{Hard graft}

Cytograft Tissue Engineering, a company based in Novato, California, is taking an alternative approach. The company's scientists have grown sheets of tissue from human fibroblast cells taken from skin biopsies. They roll the sheets into tubes around stainless-steel cylinders and leave them to grow for several weeks, until the sheets fuse into their tubular shape. After removing the cylinders, they seed dog or rat endothelial cells on the inside of the tube to generate the vessel's inner lining. Finally, they expose the vessel to varying fluid flows and pressure to prepare them for implantation. In unpub-

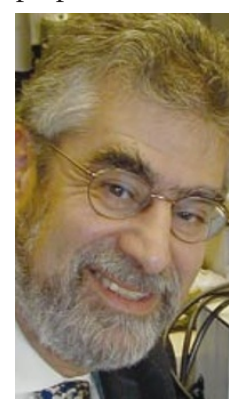

Michael Sefton: results in ten years? lished work, Cytograft scientists have grafted the resulting vessels into both dogs and rats, where they seemed to function well.

Heart valves are especially tricky to grow because of their complex architecture of cells and extracellular matrix that continuously remodels itself to respond to fluid flow in the heart. Tissue- engineered versions would be a boon for children who are born with defective valves, because mechanical implants must be successively replaced as the heart grows. A far better solution, in cases in which the problem is identified during pregnancy, would be to take a sample of fetal tissue and grow valves that would be ready for implantation shortly after birth, and which would subsequently grow along with the heart. To that end, John Mayer and his colleagues at the Children's Hospital Boston have seeded a biodegradable scaffold with endothelial and muscle cells taken from arteries in the necks of lambs. Again, the researchers pulsed fluid through the growing tissue to mimic the conditions experienced by a working valve. After implantation into the same lambs from which the cells were originally taken, the valves continued to grow, and survived for up to five months ${ }^{8}$.

Once valves, muscle, coronary blood vessels and other miscellaneous bits are constructed, the final step will be fitting the parts together into a functioning organ. Rather than growing each component separately and then tacking them together, the best approach would be to grow them all at the same time in a complex, multi-chamber bioreactor. But that's easier said than done. "The challenge will be to get them to grow in a coordinated fashion," says Frederick Schoen of the Brigham and Women's Hospital in Boston.

For the time being, it's a challenge that no one knows how to meet. But even if it proves impossible, tissue-engineered heart components might allow many patients who would otherwise need a replacement heart to carry on living without a transplant, thanks to careful running repairs. "Tissue engineering could allow damaged areas of the heart to be healed as the damage occurs," says Margaret Allen, a heart-transplant surgeon at the Hope Heart Institute, and a member of the BEAT team.

In retrospect, Sefton concedes that LIFE's ten-year timescale was unrealistic. "We were trying to capture the attention of the public," he admits. He now thinks that it may be 25 years before a patient receives a lab-grown heart, and refuses to speculate on the price tag. But he is still pursuing funding, and remains convinced that the project could produce tangible results within ten years. "We could probably have something that could work crudely," Sefton claims. "Not something ready to transplant into patients, but something you could hold in your hand."

Catherine Zandonella is a science writer in New York.

1. Hubbell, J. A. Curr. Opin. Biotechnol. 10, 123-129 (1999).

2. Keeley, F. W., Bellingham, C. M. \& Woodhouse, K. A. Phil. Trans. R. Soc. Lond. B 357, 185-189 (2002).

3. Shimizu, T. et al. Circ. Res. 90, e40-e48 (2002).

4. Radisic, M. et al. Biotechnol. Bioeng. 82, 403-414 (2003).

5. Fink, C. et al. FASEB J. 14, 669-679 (2000).

6. Eschenhagen, T., Didié, M., Heubach, J., Ravens, U. \&

Zimmermann, W.-H. Transplant Immunol. 9, 315-321 (2002).

7. Niklason, L. E. et al. Science 284, 489-493 (1999).

8. Hoerstrup, S. P. et al. Circulation 102 (suppl. III), 44-49 (2000). 